\title{
An LQP-SQP alternating direction method for solving variational inequality problems with separable structure
}

\author{
Adnan Alhomaidan ${ }^{\mathrm{a}}$, Abdellah Bnouhachem ${ }^{\mathrm{b}}$, Abdul Latifa,* \\ ${ }^{a}$ Department of Mathematics, King Abdulaziz University, P. O. Box 80203, Jeddah 21589, Saudi Arabia. \\ ${ }^{b}$ Laboratoire d'Ingnierie des Systmes et Technologies de l'Information, Ibn Zohr University, Agadir, BP 1136, Morocco. \\ Communicated by M. Bohner
}

\begin{abstract}
In this paper, by combining the logarithmic-quadratic proximal (LQP) method and the square quadratic proximal (SQP) method, we propose an inexact alternating direction method for solving constrained variational inequalities VI $(S, f)$, where $S$ is a convex set with linear constraints. Under certain conditions, the global convergence of the proposed method is established. We show the $\mathrm{O}(1 / \mathrm{t})$ convergence rate for the inexact LQP-SQP alternating direction method. To demonstrate the efficiency of the proposed method, we provide numerical results for traffic equilibrium problems. (c)2017 All rights reserved.
\end{abstract}

Keywords: Proximal point algorithm, logarithmic-quadratic proximal method, square quadratic proximal, variational inequality, prediction-correction, traffic equilibrium problems.

2010 MSC: 90C33, 49J405.

\section{Introduction}

Let $\mathbb{R}$ be the set of real numbers, and $\mathbb{R}_{+}=\{x \in \mathbb{R} ; x \geqslant 0\}, \mathbb{R}_{++}=\{x \in \mathbb{R} ; x>0\}$. Further, given $n \in \mathbb{N}$, set

$$
\mathbb{R}_{+}^{n}=\left\{x=\left(x_{1}, \cdots, x_{n}\right)^{\top}: x_{1}, \cdots, x_{n} \in \mathbb{R}_{+}\right\}
$$

and

$$
\mathbb{R}_{++}^{\mathrm{n}}=\left\{x=\left(x_{1}, \cdots, x_{\mathrm{n}}\right)^{\top}: x_{1}, \cdots, x_{\mathrm{n}} \in \mathbb{R}_{++}\right\},
$$

where $(\cdot)^{\top}$ denotes the transpose. We denote by $\mathrm{P}_{\mathbb{R}_{+}^{m}}(\cdot)$ the projection under $\mathbb{R}_{+}^{m}$, that is,

$$
\mathrm{P}_{\mathbb{R}_{+}^{m}}(z)=\operatorname{argmin}\left\{\|z-x\|: x \in \mathbb{R}_{+}^{m}\right\} .
$$

Let $A \in \mathbb{R}^{n \times m}, b \in \mathbb{R}^{m}$ and $f: \mathbb{R}_{+}^{n} \longrightarrow \mathbb{R}^{n}$ be a continuous mapping. In this paper we focus essentially on the structured variational inequalities:

$$
\text { find } x^{*} \in S \text { such that }\left(x-x^{*}\right)^{\top} f\left(x^{*}\right) \geqslant 0, \quad \forall x \in S \text {, }
$$

\footnotetext{
*Corresponding author

Email address: alatif@kau. edu.sa (Abdul Latif)
}

doi:10.22436/jnsa.010.12.10 
with linear constraints

$$
S=\left\{x \in \mathbb{R}^{n} \mid A^{\top} x \leqslant b, x \geqslant 0\right\} .
$$

By attaching the Lagrangian multiplier vector $y \in \mathbb{R}_{+}^{m}$ to the linear constraints $A^{\top} x-b \leqslant 0$, we obtain

$$
(\operatorname{VI}(\Omega, F)) \text { find } u^{*} \in \Omega \text { such that }\left(u-u^{*}\right)^{\top} F\left(u^{*}\right) \geqslant 0, \quad \forall u \in \Omega \text {, }
$$

where

$$
u=\left(\begin{array}{c}
x \\
y
\end{array}\right), \quad F(u)=\left(\begin{array}{c}
f(x)+A y \\
-A^{\top} x+b
\end{array}\right) \quad \text { and } \quad \Omega=\mathbb{R}_{+}^{n} \times \mathbb{R}_{+}^{m} .
$$

The alternating direction method (ADM) was studied extensively in optimization and variational analysis and is an attractive approach for solving large-scale variational inequality problems with separable structure, see for example $[1,5,9,12,13,16-21,24,25]$ and references therein. The classical proximal alternating directions method (PADM) is one of the most attractive ADMs, as given $\left(x^{k}, y^{k}\right) \in \Omega, x^{k+1}$ is produced via solving the following problem:

$$
x \in \mathbb{R}_{+}^{n}, \quad\left(x^{\prime}-x\right)^{\top}\left\{f(x)-A^{\top}\left[\lambda^{k}-\beta(A x-b)\right]+\left(x-x^{k}\right)\right\} \geqslant 0, \quad \forall x^{\prime} \in \mathbb{R}_{+}^{n},
$$

and the multiplier vector $y^{k+1}$ is obtained by solving the following problem:

$$
y \in \mathbb{R}_{+}^{m}, \quad\left(y^{\prime}-y\right)^{\top}\left\{\left(y-y^{k}\right)-\frac{1}{v} \beta_{k}\left(A^{\top} x^{k}-b\right)\right\} \geqslant 0, \quad \forall y^{\prime} \in \mathbb{R}_{+}^{m} .
$$

Here $v>0$ is a constant and $\beta_{k}>0$ is a given penalty parameter for the linearly constrained equation $A x-b=0$.

Recently, a significant number of interior proximal methods have been developed via replacing $x-x^{k}$ by some nonlinear functionals. Very recently, some ADMs with logarithmic-quadratic proximal (LQP) regularization $[3-8,15,22,23,25]$ have been developed by substituting in the ADM (1.3) the term $\left[\left(x-x^{k}\right)\right.$ by $\left.\left(x-x^{k}\right)+\mu\left(u^{k}-u_{k}^{2} u^{-1}\right)\right]$. He et al. [15] proposed an LQP-based prediction-correction method to solve $\operatorname{VI}(\Omega, F)$, as given $u^{k}=\left(x^{k}, y^{k}\right) \in \mathbb{R}_{++}^{n} \times \mathbb{R}_{+}^{m}$, and $\mu \in(0,1)$, the predictor $\tilde{u}^{k}=\left(\tilde{x}^{k}, \tilde{y}^{k}\right)$ is obtained via solving the following system:

$$
\left\{\begin{array}{l}
\beta_{k}(f(x)+A y)+x-x^{k}+\mu\left(x^{k}-X_{k}^{2} x^{-1}\right)=: \xi_{x}^{k} \approx 0 \\
y=P_{\mathbb{R}_{+}^{m}}\left[y^{k}+\frac{1}{v} \beta_{k}\left(A^{\top} x^{k}-b\right)\right]
\end{array}\right.
$$

where $X_{k}=\operatorname{diag}\left(x_{1}^{k}, x_{2}^{k}, \cdots, x_{n}^{k}\right), x^{-1}$ is an $n$-vector whose $j$-th element is $1 / x_{j}$, and $v>0$. The new iterate $u^{k+1}=\left(x^{k+1}, y^{k+1}\right)$ is obtained via solving the following system:

$$
\left\{\begin{array}{l}
\tau \beta_{k}\left(f\left(\tilde{x}^{k}\right)+A \tilde{y}^{k}\right)+x-x^{k}+\mu\left(x^{k}-X_{k}^{2} x^{-1}\right)=0 \\
y=P_{\mathbb{R}_{+}^{m}}\left[y^{k}+\frac{\tau}{v} \beta_{k}\left(A^{\top} \tilde{x}^{k}-b\right)\right]
\end{array}\right.
$$

Later, by applying the LQP terms to regularize the ADM subproblems, Fu and Bnouhachem [11] developed the following LQP-based decomposition method: given $u^{k}=\left(x^{k}, y^{k}\right) \in \mathbb{R}_{++}^{m} \times \mathbb{R}_{++}^{n}, \tilde{u}^{k}=\left(\tilde{x}^{k}, \tilde{y}^{k}\right)$ is obtained via solving the following system:

$$
\left\{\begin{array}{l}
\beta_{k}(f(x)+A y)+x-(1-\mu) x^{k}-\mu X_{k}^{2} x^{-1}=: \xi_{x}^{k} \approx 0, \\
\beta_{k}\left(-A^{\top} x+b\right)+v y-v(1-\mu) y^{k}-v \mu Y_{k}^{2} y^{-1}=: \xi_{y}^{k} \approx 0,
\end{array}\right.
$$

where $X_{k}=\operatorname{diag}\left(x_{1}^{k}, x_{2}^{k}, \cdots, x_{n}^{k}\right), Y_{k}=\operatorname{diag}\left(y_{1}^{k}, y_{2}^{k}, \cdots, y_{m}^{k}\right), x^{-1}\left(y^{-1}\right)$ is an $n$-vector (m-vector) whose $j$-th element is $1 / x_{j}\left(1 / y_{j}\right), \mu \in(0,1)$. The new iterate $u^{k+1}=\left(x^{k+1}, y^{k+1}\right)$ is the solution of the following system:

$$
\left\{\begin{array}{l}
\tau \beta_{k}\left(f\left(\tilde{x}^{k}\right)+A \tilde{y}^{k}\right)+x-(1-\mu) x^{k}-\mu X_{k}^{2} x^{-1}=0 \\
\tau \beta_{k}\left(-A^{\top} \tilde{x}^{k}+b\right)+v y-v(1-\mu) y^{k}-\mu \nu Y_{k}^{2} y^{-1}=0 .
\end{array}\right.
$$


Note that in [11] $\tilde{u}^{k}$ and $u^{k+1}$ are obtained via solving the LQP system. Very recently He et al. [14] proposed a new method for solving $\operatorname{VI}(\Omega, F)$, the predictor $\tilde{u}^{k}=\left(\tilde{x}^{k}, \tilde{y}^{k}\right)$ is obtained as in (1.5) and the new iterate $u^{k+1}=\left(x^{k+1}, y^{k+1}\right)$ is defined by

$$
u^{k+1}=t u^{k}+(1-t) P_{\Omega}\left[u^{k}-\alpha F\left(\tilde{u}^{k}\right)\right]
$$

where $0<\mathrm{t}<1$ and $\alpha_{k}$ is defined by

$$
\alpha_{k}=\frac{\left(u^{k}-\tilde{u}^{k}\right)^{\top} F\left(\tilde{u}^{k}\right)}{\| F\left(\tilde{u}^{k} \|^{2}\right.} .
$$

In this paper, we suggest that the complementarity subproblems arising in ADM (1.3) and (1.4) could be regularized by LQP and the square quadratic proximal (SQP) regularizations, respectively; the LQP and SQP regularizations force the solutions of ADM subproblems to be interior points of $\mathbb{R}_{+}^{n}$ and $\mathbb{R}_{+}^{\mathrm{m}}$, respectively. More specifically, the iterative scheme of ADM with LQP and SQP regularizations is as follows: given $u^{k}=\left(x^{k}, y^{k}\right) \in \mathbb{R}_{++}^{n} \times \mathbb{R}_{++}^{m}$, the solution of (1.3)-(1.4) is obtained via solving the following system:

$$
\begin{gathered}
\beta_{k}(f(x)+A y)+x-(1-\mu) x^{k}-\mu X_{k}^{2} x^{-1}=0 \\
\beta_{k}\left(-A^{\top} x+b\right)+\frac{v}{2} y-\frac{v}{2}(1-2 \mu) y^{k}-v \mu Y_{k}(\sqrt{y})^{-1}=0
\end{gathered}
$$

where $X_{k}=\operatorname{diag}\left(x_{1}^{k}, x_{2}^{k}, \cdots, x_{n}^{k}\right), Y_{k}=\operatorname{diag}\left(y_{1}^{k} \sqrt{y_{1}^{k}}, \cdots, y_{n}^{k} \sqrt{y_{m}^{k}}\right), x^{-1}\left((\sqrt{y})^{-1}\right)$ is an $n$-vector (m-vector) whose $j$-th element is $1 / x_{j}\left(1 / \sqrt{y_{j}}\right)$. Since (1.7) includes both square and quadratic terms, the method is called the SQP method, and (1.7) is called the SQP system of nonlinear equations. Note that the system (1.6)-(1.7) is a nonlinear system of equations, which is not easy to solve. Moreover, $x$ and $y$ are overlapped and should be solved simultaneously. By combining the LQP and SQP methods, we propose an inexact $\mathrm{ADM}$ for solving $\mathrm{VI}(\Omega, \mathrm{F})$. Each iteration of the proposed method contains a prediction and a correction, the predictor is obtained via solving the LQP and SQP systems approximately under significantly relaxed accuracy criterion. We also study the global convergence of the proposed method under certain conditions. Our results can be viewed as significant extensions of the previously known results.

We give some notation that is needed for the rest of the paper. Let $G$ denote a symmetric positivedefinite matrix. Then $\|v\|_{\mathrm{G}}$ denotes $\left(v^{\top} \mathrm{G} v\right)^{1 / 2}$.

Throughout this paper we make the following standard assumptions.

\section{Assumption A.}

A1. $f(x)$ is continuous and monotone mapping with respect to $\mathbb{R}_{+}^{n}$, i.e.,

$$
(x-\tilde{x})^{\top}(f(x)-f(\tilde{x})) \geqslant 0, \quad \forall x, \tilde{x} \in \mathbb{R}_{+}^{n} .
$$

A2. The solution set of $\operatorname{VI}(\Omega, F)$, denoted by $\Omega^{*}$, is nonempty. Note that $F(u)$ is monotone whenever $f(x)$ is monotone.

\section{LQP-SQP ADM}

In this section, we suggest and consider the inexact LQP-SQP ADM for solving (1.1)-(1.2). Indeed, finding the root of the nonlinear equation (1.6)-(1.7) is not an easy task. To handle this, we prefer solving 
(1.6)-(1.7) approximately to solving it exactly. First, the variable $x$ is replaced by the current $x^{k}$ in (1.7) to obtain $y$, denoted by $\tilde{y}^{k}$. Then, we find $\tilde{y}^{k} \in \mathbb{R}_{++}^{m}$ such that

$$
\beta_{k}\left(-A^{\top} x^{k}+b\right)+\frac{v}{2} \tilde{y}^{k}-\frac{v}{2}(1-2 \mu) y^{k}-v \mu Y_{k}\left(\sqrt{\tilde{y}^{k}}\right)^{-1}=0 .
$$

To use the new information as soon as possible, we use this new $\tilde{y}^{k}$ in (1.6) to obtain an approximation of $x$, denoted by $\tilde{x}^{k}$.

$$
\beta_{k}\left(f\left(x^{k}\right)+A \tilde{y}^{k}\right)+\tilde{x}^{k}-(1-\mu) x^{k}-\mu X_{k}^{2}\left(\tilde{x}^{k}\right)^{-1}=0 .
$$

We describe the new method in detail.

Algorithm 2.1 (LQP-SQP method).

Step 0. The initial step: Given $\varepsilon>0, \mu \in(0,1), \eta \in(0,1)$ and $k=0, u^{0}=\left(x^{0}, y^{0}\right) \in \mathbb{R}_{++}^{n} \times \mathbb{R}_{++}^{m}$, $v_{0}>0, \beta_{0}>0$.

Step 1. Convergence verification: If $\left\{\max \left\|x^{k}-\tilde{x}^{k}\right\|_{\infty},\left\|y^{k}-\tilde{y}^{k}\right\|_{\infty}\right\}<\epsilon$, then stop. Otherwise, go to Step 2.

Step 2. Prediction step:

(1) Compute the predictor $\tilde{u}^{k}=\left(\tilde{x}^{k}, \tilde{y}^{k}\right) \in \mathbb{R}_{++}^{n} \times \mathbb{R}_{++}^{m}$ via solving the following system

$$
\left\{\begin{array}{l}
\beta_{k}(f(x)+A y)+x-x^{k}+\mu\left(x^{k}-X_{k}^{2} x^{-1}\right)=: \xi_{x}^{k} \approx 0, \\
\beta_{k}\left(-A^{\top} x+b\right)+\frac{v_{k}}{2}\left(y-y^{k}\right)+v_{k} \mu\left(y^{k}-Y_{k}(\sqrt{y})^{-1}\right)=: \xi_{y}^{k} \approx 0,
\end{array}\right.
$$

satisfying

$$
\left\|G^{-1} \xi^{k}\right\|_{G}^{2} \leqslant \frac{1-\mu}{1+\mu} \eta^{2}\left\|u^{k}-\tilde{u}^{k}\right\|_{G}^{2}
$$

where

$$
\xi^{k}=\left(\begin{array}{c}
\xi_{x}^{k} \\
\xi_{y}^{k}
\end{array}\right)
$$

and

$$
G=\left(\begin{array}{cc}
(1+\mu) I_{n} & 0 \\
0 & \frac{v_{k}(1+\mu)}{2} I_{m}
\end{array}\right)
$$

(2) Compute $r_{k}=\sqrt{\frac{\left\|G^{-1} \xi^{k}\right\|_{G}^{2}}{\frac{1-\mu}{1+\mu}\left\|u^{k}-\tilde{u}^{k}\right\|_{G}^{2}}}$.

(3) If $r_{k}>\eta$, then reduce $\beta_{k}$ by $\beta_{k}:=\beta_{k} * 0.8 / r_{k}$ and go to Step 1 .

Step 3. Adjust $\beta$ and $v$ for the next iteration if necessary:

(1) Prepare an enlarged $\beta$ for the next iteration if $r_{k}$ is too small,

$$
\beta_{k+1}:=\left\{\begin{array}{lr}
\beta_{k} * 0.7 / r_{k} & \text { if } r_{k} \leqslant 0.5, \\
\beta_{k} & \text { otherwise. }
\end{array}\right.
$$

(2) Adjust $v$ for balancing the next iteration

$$
v_{k+1}:=\left\{\begin{array}{ll}
v_{k} * 0.5 & \text { if } t_{1}>4 t_{2}, \\
v_{k} * 2 & \text { if } t_{2}>4 t_{1}, \\
v_{k}, & \text { otherwise }
\end{array} \text { where } t_{1}:=\frac{\left\|\xi_{x}^{k}\right\|}{\sqrt{1+\mu}}, t_{2}:=\frac{\left\|\xi_{y}^{k}\right\|}{\sqrt{v_{k}}}\right.
$$

Step 4. Correction step:

Compute the new iterate $u^{k+1}\left(\alpha_{k}\right)$ as the solution of the following system

$$
\left\{\begin{array}{l}
\frac{1-\mu}{1+\mu} \alpha_{k} \beta_{k}\left(f\left(\tilde{x}^{k}\right)+A \tilde{y}^{k}\right)+x-x^{k}+\mu\left(x^{k}-X_{k}^{2} x^{-1}\right)=0 \\
\frac{1-\mu}{1+\mu} \alpha_{k} \beta_{k}\left(-A^{\top} \tilde{x}^{k}+b\right)+\frac{v_{k}}{2}\left(y-y^{k}\right)+v_{k} \mu\left(y^{k}-Y_{k}(\sqrt{y})^{-1}\right)=0 .
\end{array}\right.
$$


Set $k:=k+1$ and go to Step 1.

How to choose a suitable step length $\alpha_{k}>0$ to force convergence will be discussed later.

Remark 2.2. The main task of the prediction is to find an approximate solution of the following equations

$$
\begin{gathered}
\beta_{k}\left(f(x)+A \tilde{y}^{k}\right)+x-(1-\mu) x^{k}-\mu X_{k}^{2} x^{-1}=0, \\
\beta_{k}\left(-A^{\top} \tilde{x}^{k}+b\right)+\frac{\nu_{k}}{2}\left(y-y^{k}\right)+\nu_{k} \mu\left(y^{k}-Y_{k}(\sqrt{y})^{-1}\right)=0 .
\end{gathered}
$$

We can choose a suitable $\beta_{k}>0$ and set the exact solution of

$$
\beta_{k}\left(-A^{\top} x^{k}+b\right)+\frac{v_{k}}{2}\left(y-y^{k}\right)+v_{k} \mu\left(y^{k}-Y_{k}(\sqrt{y})^{-1}\right)=0
$$

denoted by $\tilde{y}^{k}$, as the approximate solution of (2.6). Then set the exact solution of

$$
\beta_{k}\left(f\left(x^{k}\right)+A \tilde{y}^{k}\right)+x-(1-\mu) x^{k}-\mu X_{k}^{2} x^{-1}=0,
$$

denoted by $\tilde{x}^{k}$, as the approximate solution of (2.5). It follows from (2.1) and (2.7)-(2.8) that

$$
\xi^{k}=\left(\begin{array}{c}
\xi_{x}^{k} \\
\xi_{y}^{k}
\end{array}\right)=\left(\begin{array}{c}
\beta_{k}\left(f\left(\tilde{x}^{k}\right)-f\left(x^{k}\right)\right) \\
\beta_{k}\left(-A^{\top} \tilde{x}^{k}+A^{\top} x^{k}\right)
\end{array}\right)=\beta_{k}\left(\begin{array}{c}
f\left(\tilde{x}^{k}\right)-f\left(x^{k}\right) \\
A^{\top}\left(x^{k}-\tilde{x}^{k}\right)
\end{array}\right) .
$$

Remark 2.3. We suggest a self-adaptive procedure to find such a suitable small $\beta_{k}$. If $r_{k} \leqslant \eta$, the prediction $\tilde{u}^{k}=\left(\tilde{x}^{k}, \tilde{y}^{k}\right)$ is accepted; otherwise, reduce the value of $\beta_{k}$ by $\beta_{k}:=\beta_{k} * 0.8 / r_{k}$ and repeat the procedure.

Too small values of $\beta_{k}$, however, usually lead to extremely slow convergence according to our numerical experiments. Thus it is necessary to avoid this situation. In addition, balancing $\left(\left\|\xi_{x}^{k}\right\| / \sqrt{1+\mu}\right)$ and $\left(\left\|\xi_{y}^{k}\right\| / \sqrt{v_{k}}\right)$ via adjusting $v$ is also necessary for practical computation.

We need the following result to study the convergence analysis of the proposed method.

Lemma 2.4. Given $\mathrm{u}^{\mathrm{k}}=\left(\mathrm{x}^{\mathrm{k}}, \mathrm{y}^{\mathrm{k}}\right) \in \mathbb{R}_{++}^{\mathrm{n}} \times \mathbb{R}_{++}^{\mathrm{m}}$ and $\mathrm{q}=\left(\mathrm{q}_{\mathrm{x}}, \mathrm{q}_{\mathrm{y}}\right) \in \mathbb{R}^{\mathrm{n}} \times \mathbb{R}^{\mathrm{m}}$, let $\mathrm{u}=(\mathrm{x}, \mathrm{y})$ be the positive solution of the following equations:

$$
\left\{\begin{array}{l}
q_{x}+x-x^{k}+\mu\left(x^{k}-X_{k}^{2} x^{-1}\right)=0 \\
q_{y}+\frac{v_{k}}{2}\left(y-y^{k}\right)+v_{k} \mu\left(y^{k}-\gamma_{k}(\sqrt{y})^{-1}\right)=0,
\end{array}\right.
$$

where $X_{k}=\operatorname{diag}\left(x_{1}^{k}, x_{2}^{k}, \cdots, x_{n}^{k}\right), x^{-1}=\left(1 / x_{1}, \cdots, 1 / x_{n}\right), Y_{k}=\operatorname{diag}\left(y_{1}^{k} \sqrt{y_{1}^{k}}, \cdots, y_{m}^{k} \sqrt{y_{m}^{k}}\right)$ and $(\sqrt{y})^{-1}=$ $\left(\sqrt{1 / y_{1}}, \cdots, \sqrt{1 / y_{m}}\right)$. Then for any $w \in \mathbb{R}_{+}^{n} \times \mathbb{R}_{+}^{m}$, we have

$$
(w-u)^{\top} q \geqslant \frac{1}{2}\left(\|u-w\|_{G}^{2}-\left\|u^{k}-w\right\|_{G}^{2}\right)+\frac{1-\mu}{2}\left\|x^{k}-x\right\|^{2}+\frac{v_{k}(1-\mu)}{4}\left\|y^{k}-y\right\|^{2},
$$

where $\mathrm{G}$ is defined by (2.3).

Proof. Let $w=\left(w_{x}, w_{y}\right)$. We will show that

$$
\left(w_{x}-x\right)^{\top} q_{x} \geqslant \frac{1+\mu}{2}\left(\left\|x-w_{x}\right\|^{2}-\left\|x^{k}-w_{x}\right\|^{2}\right)+\frac{1-\mu}{2}\left\|x-x^{k}\right\|^{2},
$$

and

$$
\left(w_{y}-y\right)^{\top} q_{y} \geqslant \frac{v_{k}(1+\mu)}{4}\left(\left\|y-w_{y}\right\|^{2}-\left\|y^{k}-w_{y}\right\|^{2}\right)+\frac{v_{k}(1-\mu)}{4}\left\|y-y^{k}\right\|^{2} .
$$

The first inequality is inspired by [2, Lemma 2]. Since $x>0, x^{k}>0$ and $w_{x} \geqslant 0$, we have

$$
\left(w_{x}\right)_{i}\left(x_{i}^{k}\right)^{2} / x_{i} \geqslant\left(w_{x}\right)_{i}\left(2 x_{i}^{k}-x_{i}\right) .
$$


It follows from (2.9) that

$$
\begin{aligned}
\left(\left(w_{x}\right)_{i}-x_{i}\right)^{\top}\left(q_{x}\right)_{i} & =\left(x_{i}-\left(w_{x}\right)_{i}\right)\left(x_{i}-(1-\mu) x_{i}^{k}-\mu\left(x_{i}^{k}\right)^{2} / x_{i}\right) \\
& \geqslant\left(x_{i}\right)^{2}-(1-\mu) x_{i} x_{i}^{k}-\mu\left(x_{i}^{k}\right)^{2}-x_{i}\left(w_{x}\right)_{i}+(1-\mu) x_{i}^{k}\left(w_{x}\right)_{i}+\mu\left(w_{x}\right)_{i}\left(2 x_{i}^{k}-x_{i}\right) \\
& =\left(x_{i}\right)^{2}-(1-\mu) x_{i} x_{i}^{k}-\mu\left(x_{i}^{k}\right)^{2}-(1+\mu) x_{i}\left(w_{x}\right)_{i}+(1+\mu) x_{i}^{k}\left(w_{x}\right)_{i} \\
& =\frac{1+\mu}{2}\left(\left(x_{i}-\left(w_{x}\right)_{i}\right)^{2}-\left(x_{i}^{k}-\left(w_{x}\right)_{i}\right)^{2}\right)+\frac{1-\mu}{2}\left(x_{i}^{k}-x_{i}\right)^{2} .
\end{aligned}
$$

Hence, (2.11) holds.

For each $t>0$ we have $\frac{1}{2}\left(1-\frac{1}{t}\right) \leqslant 1-\frac{1}{\sqrt{t}} \leqslant \frac{1}{2}(t-1)$. Since $y>0, y^{k}$ and $w_{y} \geqslant 0$, by using the above inequality, then after multiplication by $\left(w_{y}\right)_{j} y_{j}^{k} \geqslant 0$ for each $j=1, \cdots, m$, we obtain

$$
\left(w_{y}\right)_{j} y_{j}^{k}\left(1-\frac{\sqrt{y_{j}^{k}}}{\sqrt{y_{j}}}\right) \leqslant\left(w_{y}\right)_{j} y_{j}^{k} \frac{1}{2}\left(\frac{y_{j}}{y_{j}^{k}}-1\right)=\frac{1}{2}\left(w_{y}\right)_{j}\left(y_{j}-y_{j}^{k}\right),
$$

and after multiplication by $y_{j} y_{j}^{k} \geqslant 0$ for each $j=1, \cdots, m$,

$$
-y_{j} y_{j}^{k}\left(1-\frac{\sqrt{y_{j}^{k}}}{\sqrt{y_{j}}}\right) \leqslant y_{j} y_{j}^{k} \frac{1}{2}\left(\frac{y_{j}^{k}}{y_{j}}-1\right)=\frac{1}{2} y_{j}^{k}\left(y_{j}^{k}-y_{j}\right),
$$

adding the two inequalities, then we obtain

$$
\begin{aligned}
\left(\left(w_{y}\right)_{j}-y_{j}\right) & \left(\frac{1}{2} v_{k}\left(y_{j}-y_{j}^{k}\right)+\mu v_{k}\left(y_{j}^{k}-y_{j}^{k} \sqrt{y_{j}^{k}}\left(\sqrt{y_{j}}-1\right)\right)\right. \\
& \leqslant \frac{1}{2} v_{k} \mu\left(\left(w_{y}\right)_{j}-y_{j}^{k}\right)\left(y_{j}-y_{j}^{k}\right)+\frac{1}{2} v_{k}\left(y_{j}-y_{j}^{k}\right)\left(\left(w_{y}\right)_{j}-y_{j}\right) .
\end{aligned}
$$

Using the identities

$$
\begin{aligned}
& \frac{1}{2}\left(\left(w_{y}\right)_{j}-y_{j}^{k}\right)\left(y_{j}-y_{j}^{k}\right)=\frac{1}{4}\left(\left(y_{j}-y_{j}^{k}\right)^{2}-\left(\left(w_{y}\right)_{j}-y_{j}\right)^{2}+\left(\left(w_{y}\right)_{j}-y_{j}^{k}\right)^{2}\right), \\
& \frac{1}{2}\left(y_{j}-y_{j}^{k}\right)\left(\left(w_{y}\right)_{j}-y_{j}\right)=\frac{1}{4}\left(\left(\left(w_{y}\right)_{j}-y_{j}^{k}\right)^{2}-\left(\left(w_{y}\right)_{j}-y_{j}\right)^{2}-\left(y_{j}-y_{j}^{k}\right)^{2}\right),
\end{aligned}
$$

and recalling (2.9), thus we obtain

$$
\left(\left(w_{y}\right)_{j}-y_{j}\right)\left(q_{y}\right)_{j} \geqslant \frac{v_{k}(1+\mu)}{4}\left(\left(\left(w_{y}\right)_{j}-y_{j}\right)^{2}-\left(\left(w_{y}\right)_{j}-y_{j}^{k}\right)^{2}\right)+\frac{v_{k}(1-\mu)}{4}\left(y_{j}^{k}-y_{j}\right)^{2} .
$$

Summing over $j=1, \cdots, n$, we obtain (2.12). Adding (2.11) and (2.12) the proof is complete.

\section{Basic results}

In this section, we prove some basic properties, that will be used to establish the sufficient and necessary conditions for the convergence of the proposed method. First, we apply Lemma 2.4 to the prediction step.

Lemma 3.1. Given $\mathrm{u}^{k}=\left(x^{k}, y^{k}\right) \in \mathbb{R}_{++}^{n} \times \mathbb{R}_{++}^{m}$, let $\tilde{u}^{k}$ be the predictor produced by (2.1). For each $w=$ $\left(w_{x}, w_{y}\right) \in \Omega$, we have

$$
\begin{aligned}
\left(w-\tilde{u}^{k}\right)^{\top}\left(\xi^{k}-\beta_{k} F\left(\tilde{u}^{k}\right)\right) \leqslant & \frac{1}{2}\left(\left\|u^{k}-w\right\|_{G}^{2}\right. \\
& \left.-\left\|\tilde{u}^{k}-w\right\|_{G}^{2}\right)-\frac{1-\mu}{2}\left\|x^{k}-\tilde{x}^{k}\right\|^{2}-\frac{v_{k}(1-\mu)}{4}\left\|y^{k}-\tilde{y}^{k}\right\|^{2}
\end{aligned}
$$


Proof. We apply Lemma 2.4 to the prediction equation (2.1). Note that

$$
q_{\text {pre }}:=\left(\begin{array}{c}
\beta_{k}\left(f\left(\tilde{x}^{k}\right)+A \tilde{y}^{k}\right)-\xi_{x}^{k} \\
\beta_{k}\left(-A^{\top} \tilde{x}^{k}+b\right)-\xi_{y}^{k}
\end{array}\right)=\beta_{k} F\left(\tilde{u}^{k}\right)-\xi^{k} .
$$

By setting $q=q_{\text {pre }}$ in (2.9) and $u=\tilde{u}^{k}$ in (2.10), it follows that

$$
\left(w-\tilde{u}^{k}\right)^{\top}\left(-q_{\text {pre }}\right) \leqslant \frac{1}{2}\left(\left\|u^{k}-w\right\|_{G}^{2}-\left\|\tilde{u}^{k}-w\right\|_{G}^{2}\right)-\frac{1-\mu}{2}\left\|x^{k}-\tilde{x}^{k}\right\|^{2}-\frac{v_{k}(1-\mu)}{4}\left\|y^{k}-\tilde{y}^{k}\right\|^{2} .
$$

The assertion is proved.

Now, we apply Lemma 2.4 to the correction step.

Lemma 3.2. Given $\mathrm{u}^{\mathrm{k}}=\left(\mathrm{x}^{\mathrm{k}}, \mathrm{y}^{\mathrm{k}}\right) \in \mathbb{R}_{++}^{\mathrm{n}} \times \mathbb{R}_{++}^{\mathrm{m}}$, let $\tilde{\mathrm{u}}^{\mathrm{k}}$ be the predictor produced by (2.1) and $\mathrm{u}^{\mathrm{k}+1}\left(\alpha_{\mathrm{k}}\right)$ be the corrector produced by (2.4). Then, we have

$$
\begin{aligned}
\left\|u^{k}-u^{*}\right\|_{G}^{2}-\left\|u^{k+1}\left(\alpha_{k}\right)-u^{*}\right\|_{G}^{2} \geqslant & (1-\mu)\left\|x^{k}-x^{k+1}\left(\alpha_{k}\right)\right\|^{2}+\frac{v_{k}(1-\mu)}{2}\left\|y^{k}-y^{k+1}\left(\alpha_{k}\right)\right\|^{2} \\
& +2 \frac{1-\mu}{1+\mu} \alpha_{k} \beta_{k} F\left(\tilde{u}^{k}\right)^{\top}\left(u^{k+1}\left(\alpha_{k}\right)-\tilde{u}^{k}\right) .
\end{aligned}
$$

Proof. The proof is an application of Lemma 2.4 to the correction equation (2.2).

$$
q_{\text {cor }}:=\left(\begin{array}{c}
\frac{1-\mu}{1+\mu} \alpha_{k} \beta_{k}\left(f\left(\tilde{x}^{k}\right)+A \tilde{y}^{k}\right) \\
\frac{1-\mu}{1+\mu} \alpha_{k} \beta_{k}\left(-A^{\top} \tilde{x}^{k}+b\right)
\end{array}\right)=\frac{1-\mu}{1+\mu} \alpha_{k} \beta_{k} F\left(\tilde{u}^{k}\right) .
$$

By setting $\mathrm{q}=\mathrm{q}_{\mathrm{cor}}$ in (2.9) and $w=\mathrm{u}^{*} \in \Omega^{*}, \mathrm{u}=\mathrm{u}^{\mathrm{k}+1}\left(\alpha_{\mathrm{k}}\right)$ in (2.10), we obtain

$$
\begin{aligned}
\left(u^{*}-u^{k+1}\left(\alpha_{k}\right)\right)^{\top} q_{c o r} \geqslant & \frac{1}{2}\left(\left\|u^{k+1}\left(\alpha_{k}\right)-u^{*}\right\|_{G}^{2}-\left\|u^{k}-u^{*}\right\|_{G}^{2}\right) \\
& +\frac{1-\mu}{2}\left\|x^{k}-x^{k+1}\left(\alpha_{k}\right)\right\|^{2}+\frac{v_{k}(1-\mu)}{4}\left\|y^{k}-y^{k+1}\left(\alpha_{k}\right)\right\|^{2} .
\end{aligned}
$$

It follows from the above inequality that

$$
\begin{aligned}
\left\|u^{k}-u^{*}\right\|_{G}^{2}-\left\|u^{k+1}\left(\alpha_{k}\right)-u^{*}\right\|_{G}^{2} \geqslant & (1-\mu)\left\|x^{k}-x^{k+1}\left(\alpha_{k}\right)\right\|^{2}+\frac{v_{k}(1-\mu)}{2}\left\|y^{k}-y^{k+1}\left(\alpha_{k}\right)\right\|^{2} \\
& +2 \frac{1-\mu}{1+\mu} \alpha_{k} \beta_{k} F\left(\tilde{u}^{k}\right)^{\top}\left(u^{k+1}\left(\alpha_{k}\right)-u^{*}\right) \\
= & (1-\mu)\left\|x^{k}-x^{k+1}\left(\alpha_{k}\right)\right\|^{2}+\frac{v_{k}(1-\mu)}{2}\left\|y^{k}-y^{k+1}\left(\alpha_{k}\right)\right\|^{2} \\
& \times 2 \frac{1-\mu}{1+\mu} \alpha_{k} \beta_{k} F\left(\tilde{u}^{k}\right)^{\top}\left\{\left(u^{k+1}\left(\alpha_{k}\right)-\tilde{u}^{k}\right)+\left(\tilde{u}^{k}-u^{*}\right)\right\} \\
\geqslant & (1-\mu)\left\|x^{k}-x^{k+1}\left(\alpha_{k}\right)\right\|^{2}+\frac{v_{k}(1-\mu)}{2}\left\|y^{k}-y^{k+1}\left(\alpha_{k}\right)\right\|^{2} \\
& +2 \frac{1-\mu}{1+\mu} \alpha_{k} \beta_{k} F\left(\tilde{u}^{k}\right)^{\top}\left(u^{k+1}\left(\alpha_{k}\right)-\tilde{u}^{k}\right) .
\end{aligned}
$$

The last inequality of (3.3) uses the fact that $\mathrm{F}$ is monotone and the inequality

$$
\left(\tilde{u}^{k}-u^{*}\right)^{\top}\left(F\left(\tilde{u}^{k}\right)\right) \geqslant\left(\tilde{u}^{k}-u^{*}\right)^{\top} F\left(u^{*}\right) \geqslant 0 .
$$

Consequently, we have the following theorem. 
Theorem 3.3. Given $\mathrm{u}^{\mathrm{k}}=\left(\mathrm{x}^{\mathrm{k}}, \mathrm{y}^{\mathrm{k}}\right) \in \mathbb{R}_{++}^{\mathrm{n}} \times \mathbb{R}_{++}^{\mathrm{m}}$, let $\tilde{\mathrm{u}}^{\mathrm{k}}$ be the predictor produced by (2.1) and $\mathrm{u}^{\mathrm{k}+1}\left(\alpha_{\mathrm{k}}\right)$ be the corrector produced by (2.4). Then, we have

$$
\begin{aligned}
\left\|u^{k}-u^{*}\right\|_{G}^{2}-\left\|u^{k+1}\left(\alpha_{k}\right)-u^{*}\right\|_{G}^{2} \geqslant & \frac{1-\mu}{1+\mu}\left(\left\|u^{k}-u^{k+1}\left(\alpha_{k}\right)\right\|_{G}^{2}+2 \alpha_{k}\left(u^{k+1}\left(\alpha_{k}\right)-\tilde{u}^{k}\right)^{\top} G d\left(u^{k}, \tilde{u}^{k}\right)\right. \\
& \left.-2 \alpha_{k} \mu\left\|x^{k}-\tilde{x}^{k}\right\|^{2}-\alpha_{k} v_{k} \mu\left\|y^{k}-\tilde{y}^{k}\right\|^{2}\right),
\end{aligned}
$$

where

$$
d\left(u^{k}, \tilde{u}^{k}\right)=\left(u^{k}-\tilde{u}^{k}\right)+G^{-1} \xi^{k} .
$$

Proof. Since $u^{k+1}\left(\alpha_{k}\right) \in \Omega$, by substituting $w=u^{k+1}\left(\alpha_{k}\right)$ in (3.1) we obtain

$$
\begin{aligned}
\left(u^{k+1}\left(\alpha_{k}\right)-\tilde{u}^{k}\right)^{\top}\left(\xi^{k}-\beta_{k} F\left(\tilde{u}^{k}\right)\right) \leqslant & \frac{1}{2}\left(\left\|u^{k}-u^{k+1}\left(\alpha_{k}\right)\right\|_{G}^{2}-\left\|\tilde{u}^{k}-u^{k+1}\left(\alpha_{k}\right)\right\|_{G}^{2}\right) \\
& -\frac{1-\mu}{2}\left\|x^{k}-\tilde{x}^{k}\right\|^{2}-\frac{v_{k}(1-\mu)}{4}\left\|y^{k}-\tilde{y}^{k}\right\|^{2} .
\end{aligned}
$$

Using the following identity

$$
\left(u^{k+1}\left(\alpha_{k}\right)-\tilde{u}^{k}\right)^{\top} G\left(u^{k}-\tilde{u}^{k}\right)=\frac{1}{2}\left(\left\|\tilde{u}^{k}-u^{k+1}\left(\alpha_{k}\right)\right\|_{G}^{2}-\left\|u^{k}-u^{k+1}\left(\alpha_{k}\right)\right\|_{G}^{2}\right)+\frac{1}{2}\left\|u^{k}-\tilde{u}^{k}\right\|_{G}^{2} .
$$

Adding (3.6) and (3.7), we obtain (using the definition of G from (2.3))

$$
\left(u^{k+1}\left(\alpha_{k}\right)-\tilde{u}^{k}\right)^{\top}\left\{G\left(u^{k}-\tilde{u}^{k}\right)+\xi^{k}-\beta_{k} F\left(\tilde{u}^{k}\right)\right\} \leqslant \mu\left\|x^{k}-\tilde{x}^{k}\right\|+\frac{v_{k} \mu}{2}\left\|y^{k}-\tilde{y}^{k}\right\|,
$$

which implies

$$
\left(u^{k+1}\left(\alpha_{k}\right)-\tilde{u}^{k}\right)^{\top} \beta_{k} F\left(\tilde{u}^{k}\right) \geqslant\left(u^{k+1}\left(\alpha_{k}\right)-\tilde{u}^{k}\right)^{\top} G d\left(u^{k}, \tilde{u}^{k}\right)-\mu\left\|x^{k}-\tilde{x}^{k}\right\|^{2}-\frac{v_{k} \mu}{2}\left\|y^{k}-\tilde{y}^{k}\right\|^{2} .
$$

Combining (3.3) and (3.8), we obtain

$$
\begin{aligned}
\left\|u^{k}-u^{*}\right\|_{G}^{2}-\left\|u^{k+1}\left(\alpha_{k}\right)-u^{*}\right\|_{G}^{2} \geqslant & (1-\mu)\left\|x^{k}-x^{k+1}\left(\alpha_{k}\right)\right\|^{2}+\frac{v_{k}(1-\mu)}{2}\left\|y^{k}-y^{k+1}\left(\alpha_{k}\right)\right\|^{2} \\
& +2 \frac{1-\mu}{1+\mu} \alpha_{k}\left(u^{k+1}\left(\alpha_{k}\right)-\tilde{u}^{k}\right)^{\top} G d\left(u^{k}, \tilde{u}^{k}\right) \\
& -2 \frac{1-\mu}{1+\mu} \alpha_{k} \mu\left\|x^{k}-\tilde{x}^{k}\right\|^{2} \\
& -\frac{1-\mu}{1+\mu} \alpha_{k} \mu v_{k}\left\|y^{k}-\tilde{y}^{k}\right\|^{2} .
\end{aligned}
$$

We observe the first two terms of the right-hand-side of (3.9),

$$
\begin{aligned}
(1-\mu) & \left\|x^{k}-x^{k+1}\left(\alpha_{k}\right)\right\|^{2}+\frac{v_{k}(1-\mu)}{2}\left\|y^{k}-y^{k+1}\left(\alpha_{k}\right)\right\|^{2} \\
= & \frac{1-\mu}{1+\mu}\left((1+\mu)\left\|x^{k}-x^{k+1}\left(\alpha_{k}\right)\right\|^{2}+\frac{v_{k}(1+\mu)}{2}\left\|y^{k}-y^{k+1}\left(\alpha_{k}\right)\right\|^{2}\right) \\
= & \frac{1-\mu}{1+\mu}\left\|u^{k}-u^{k+1}\left(\alpha_{k}\right)\right\|_{G}^{2} .
\end{aligned}
$$

Substituting it into (3.9), we obtain the required result.

Theorem 3.4. Let $\mathrm{d}\left(\mathrm{u}^{\mathrm{k}}, \tilde{u}^{\mathrm{k}}\right)$ be defined by (3.5). Then for any $\mathrm{u}^{*} \in \Omega^{*}$ and $\alpha_{\mathrm{k}}>0$, we have

$$
\left\|u^{k}-u^{*}\right\|_{G}^{2}-\left\|u^{k+1}\left(\alpha_{k}\right)-u^{*}\right\|_{G}^{2} \geqslant \frac{1-\mu}{1+\mu} \Phi\left(\alpha_{k}\right),
$$

where 


$$
\Phi\left(\alpha_{k}\right):=2 \alpha_{k} \varphi\left(u^{k}, \tilde{u}^{k}\right)-\alpha_{k}^{2}\left\|d\left(u^{k}, \tilde{u}^{k}\right)\right\|_{G}^{2},
$$

and

$$
\varphi\left(u^{k}, \tilde{u}^{k}\right):=\left\|x^{k}-\tilde{x}^{k}\right\|^{2}+\frac{v_{k}}{2}\left\|y^{k}-\tilde{y}^{k}\right\|^{2}+\left(u^{k}-\tilde{u}^{k}\right)^{\top} \xi^{k} .
$$

Proof. It follows from (3.4) and (3.5) that

$$
\begin{aligned}
\left\|u^{k}-u^{*}\right\|_{G}^{2}-\left\|u^{k+1}\left(\alpha_{k}\right)-u^{*}\right\|_{G}^{2} \geqslant & \frac{1-\mu}{1+\mu}\left(2 \alpha_{k}\left\{\left(u^{k+1}\left(\alpha_{k}\right)-u^{k}\right)+\left(u^{k}-\tilde{u}^{k}\right)\right\}^{\top} G d\left(u^{k}, \tilde{u}^{k}\right)\right. \\
& \left.-2 \alpha_{k} \mu\left\|x^{k}-\tilde{x}^{k}\right\|^{2}-\alpha_{k} v_{k} \mu\left\|y^{k}-\tilde{y}^{k}\right\|^{2}+\left\|u^{k}-u^{k+1}\left(\alpha_{k}\right)\right\|_{G}^{2}\right) \\
= & \frac{1-\mu}{1+\mu}\left(2 \alpha_{k}\left(u^{k}-\tilde{u}^{k}\right)^{\top} G d\left(u^{k}, \tilde{u}^{k}\right)-2 \alpha_{k} \mu\left\|x^{k}-\tilde{x}^{k}\right\|^{2}\right. \\
& -\alpha_{k} v_{k} \mu\left\|y^{k}-\tilde{y}^{k}\right\|^{2}-\alpha_{k}^{2}\left\|d\left(u^{k}, \tilde{u}^{k}\right)\right\|_{G}^{2} \\
& \left.+\left\|u^{k}-u^{k+1}\left(\alpha_{k}\right)-\alpha_{k} d\left(u^{k}, \tilde{u}^{k}\right)\right\|_{G}^{2}\right) \\
\geqslant & \frac{1-\mu}{1+\mu}\left(2 \alpha _ { k } \left\{\left(u^{k}-\tilde{u}^{k}\right)^{\top} G d\left(u^{k}, \tilde{u}^{k}\right)-\mu\left\|x^{k}-\tilde{x}^{k}\right\|^{2}\right.\right. \\
& \left.\left.-\frac{v_{k} \mu}{2}\left\|y^{k}-\tilde{y}^{k}\right\|^{2}\right\}-\alpha_{k}^{2}\left\|d\left(u^{k}, \tilde{u}^{k}\right)\right\|_{G}^{2}\right) \\
= & \frac{1-\mu}{1+\mu}\left(2 \alpha_{k}\left\{\left\|x^{k}-\tilde{x}^{k}\right\|^{2}+\frac{v_{k}}{2}\left\|y^{k}-\tilde{y}^{k}\right\|^{2}+\left(u^{k}-\tilde{u}^{k}\right)^{\top} \xi^{k}\right\}\right. \\
& \left.-\alpha_{k}^{2}\left\|d\left(u^{k}, \tilde{u}^{k}\right)\right\|_{G}^{2}\right) \\
= & \frac{1-\mu}{1+\mu}\left(2 \alpha_{k} \varphi\left(u^{k}, \tilde{u}^{k}\right)-\alpha^{2}\left\|d\left(u^{k}, \tilde{u}^{k}\right)\right\|_{G}^{2}\right) .
\end{aligned}
$$

The assertion follows directly from (3.10) and (3.11).

Since $\Phi\left(\alpha_{k}\right)$ is concave quadratic function of $\alpha_{k}$, it reaches its maximum at

$$
\alpha_{\mathrm{k}}^{*}=\frac{\varphi\left(\mathrm{u}^{\mathrm{k}}, \tilde{\mathrm{u}}^{\mathrm{k}}\right)}{\left\|\mathrm{d}\left(\mathrm{u}^{\mathrm{k}}, \tilde{\mathrm{u}}^{\mathrm{k}}\right)\right\|_{\mathrm{G}}^{2}},
$$

with

$$
\Phi\left(\alpha_{k}^{*}\right)=\alpha_{k}^{*} \varphi\left(u^{k}, \tilde{u}^{k}\right)
$$

Under condition (2.2) we have

$$
\begin{aligned}
2 \varphi\left(u^{k}, \tilde{u}^{k}\right)-\left\|d\left(u^{k}, \tilde{u}^{k}\right)\right\|_{G}^{2} & =2\left\|x^{k}-\tilde{x}^{k}\right\|^{2}+v_{k}\left\|y^{k}-\tilde{y}^{k}\right\|^{2}-\left\|u^{k}-\tilde{u}^{k}\right\|_{G}^{2}-\left\|G^{-1} \xi^{k}\right\|_{G}^{2} \\
& =(1-\mu)\left\|x^{k}-\tilde{x}^{k}\right\|^{2}+\frac{v_{k}(1-\mu)}{2}\left\|y^{k}-\tilde{y}^{k}\right\|^{2}-\left\|G^{-1} \xi^{k}\right\|_{G}^{2} \\
& =\frac{1-\mu}{1+\mu}\left((1+\mu)\left\|x^{k}-\tilde{x}^{k}\right\|^{2}+\frac{v_{k}(1+\mu)}{2}\left\|y^{k}-\tilde{y}^{k}\right\|^{2}\right)-\left\|G^{-1} \xi^{k}\right\|_{G}^{2} \\
& =\frac{1-\mu}{1+\mu}\left\|u^{k}-\tilde{u}^{k}\right\|_{G}^{2}-\left\|G^{-1} \xi^{k}\right\|_{G}^{2} \\
& \geqslant \frac{1-\mu}{1+\mu}\left(1-\eta^{2}\right)\left\|u^{k}-\tilde{u}^{k}\right\|_{G}^{2} .
\end{aligned}
$$

Therefore, it follows from (3.12) and (3.14) that

$$
\alpha_{\mathrm{k}}^{*} \geqslant \frac{1}{2} .
$$

Consequently, from (3.13), (3.14), and (3.15), we obtain

$$
\Phi\left(\alpha_{\mathrm{k}}^{*}\right) \geqslant \frac{\left(1-\eta^{2}\right)(1-\mu)}{4(1+\mu)}\left\|u^{k}-\tilde{u}^{k}\right\|_{G}^{2} .
$$




\section{Convergence of the proposed method}

In this section, we consider the convergence analysis of the proposed method. From the numerical point of view, it is necessary to attach a relaxation factor $\gamma \in[1,2)$ to the optimal step size $\alpha_{k}$ to achieve faster convergence.

Theorem 4.1. Let $\mathrm{u}^{\mathrm{k}+1}\left(\gamma \alpha^{*}\right)$ be the solution of (2.4). Then for any $\mathrm{u}^{*} \in \Omega^{*}$ and $\gamma \in[1,2)$, we have

$$
\left\|u^{k+1}\left(\gamma \alpha^{*}\right)-u^{*}\right\|_{G}^{2} \leqslant\left\|u^{k}-u^{*}\right\|_{G}^{2}-\frac{\gamma(2-\gamma)\left(1-\eta^{2}\right)}{4}\left(\frac{1-\mu}{1+\mu}\right)^{2}\left\|u^{k}-\tilde{u}^{k}\right\|_{G}^{2} .
$$

Proof. Note that for $\gamma \in[1,2)$, we obtain

$$
\begin{aligned}
\Phi\left(\gamma \alpha_{\mathrm{k}}^{*}\right) & =2 \gamma \alpha_{\mathrm{k}}^{*} \varphi\left(\mathrm{u}^{\mathrm{k}}, \tilde{\mathrm{u}}^{\mathrm{k}}\right)-\left(\gamma^{2} \alpha_{\mathrm{k}}^{*}\right)\left(\alpha_{\mathrm{k}}^{*}\left\|\mathrm{~d}\left(\mathrm{u}^{\mathrm{k}}, \tilde{\mathrm{u}}^{\mathrm{k}}\right)\right\|_{\mathrm{G}}^{2}\right) \\
& =\left(2 \gamma \alpha_{\mathrm{k}}^{*}-\gamma^{2} \alpha_{\mathrm{k}}^{*}\right) \varphi\left(\mathrm{u}^{\mathrm{k}}, \tilde{\mathrm{u}}^{\mathrm{k}}\right) \\
& =\gamma(2-\gamma) \Phi\left(\alpha_{\mathrm{k}}^{*}\right) .
\end{aligned}
$$

It follows from Theorem 3.4 and (4.1) that

$$
\begin{aligned}
\left\|u^{k}-u^{*}\right\|^{2}-\left\|u^{k+1}\left(\gamma \alpha^{*}\right)-u^{*}\right\|^{2} & \geqslant \frac{1-\mu}{1+\mu} \Phi\left(\gamma \alpha^{*}\right) \\
& =\gamma(2-\gamma) \frac{1-\mu}{1+\mu} \Phi\left(\alpha^{*}\right) .
\end{aligned}
$$

Then the assertion follows from (3.16) immediately.

To prove the convergence of the proposed method we need the following lemma, the proof of which is again an application of Lemma 3.1.

Lemma 4.2. For given $u^{k}=\left(x^{k}, y^{k}\right) \in \mathbb{R}_{++}^{n} \times \mathbb{R}_{++}^{m}$ and $\beta_{k}>0$, let $\tilde{u}^{k}$ be obtained by (2.1), then for each $\mathrm{u} \in \Omega$, we have

$$
\left(u-\tilde{u}^{k}\right)^{\top}\left(\beta_{k} F\left(\tilde{u}^{k}\right)-\xi^{k}\right) \geqslant\left(\begin{array}{c}
x^{k}-\tilde{x}^{k} \\
y^{k}-\tilde{y}^{k}
\end{array}\right)^{\top}\left(\begin{array}{c}
(1+\mu) x-\left(\mu x^{k}+\tilde{x}^{k}\right) \\
\frac{v_{k}(1+\mu)}{2} y-\frac{v_{k}}{2}\left(\mu y^{k}+\tilde{y}^{k}\right)
\end{array}\right) .
$$

Proof. First, substituting $w=u$ in (3.1) we have

$$
\begin{aligned}
\left(u-\tilde{u}^{k}\right)^{\top}\left(\beta_{k} F\left(\tilde{u}^{k}-\xi^{k}\right)\right) \geqslant & \frac{1}{2}\left(\left\|\tilde{u}^{k}-u\right\|_{G}^{2}-\left\|u^{k}-u\right\|_{G}^{2}\right) \\
& +\frac{1-\mu}{2}\left\|x-\tilde{x}^{k}\right\|^{2}+\frac{v_{k}(1-\mu)}{4}\left\|y^{k}-\tilde{y}^{k}\right\|^{2} .
\end{aligned}
$$

Therefore, to show (4.2), we need only to prove

$$
\frac{1+\mu}{2}\left(\left\|\tilde{x}^{k}-x\right\|^{2}-\left\|x^{k}-x\right\|^{2}\right)+\frac{1-\mu}{2}\left\|x^{k}-\tilde{x}^{k}\right\|^{2}=\left(x^{k}-\tilde{\chi}^{k}\right)^{\top}\left((1+\mu) x-\left(\mu x^{k}+\tilde{x}^{k}\right)\right),
$$

and

$$
\frac{v_{k}(1+\mu)}{4}\left(\left\|\tilde{y}^{k}-y\right\|^{2}-\left\|y^{k}-y\right\|^{2}\right)+\frac{v_{k}(1-\mu)}{4}\left\|y^{k}-\tilde{y}^{k}\right\|^{2}=\frac{v_{k}}{2}\left(y^{k}-\tilde{y}^{k}\right)^{\top}\left((1+\mu) y-\left(\mu y^{k}+\tilde{y}^{k}\right)\right) \text {. }
$$

First, by a manipulation we obtain

$$
\begin{aligned}
\frac{1+\mu}{2}\left(\left\|\tilde{x}^{k}-x\right\|^{2}\right. & \left.-\left\|x^{k}-x\right\|^{2}\right)+\frac{1-\mu}{2}\left\|x^{k}-\tilde{x}^{k}\right\|^{2} \\
& =(1+\mu) x^{\top} x^{k}-(1+\mu) x^{\top} \tilde{x}^{k}-(1-\mu)\left(\tilde{x}^{k}\right)^{\top} x^{k}-\mu\left\|x^{k}\right\|^{2}+\left\|\tilde{x}^{k}\right\|^{2} \\
& =(1+\mu) x^{\top}\left(x^{k}-\tilde{x}^{k}\right)-\left(x^{k}-\tilde{x}^{k}\right)^{\top}\left(\mu x^{k}+\tilde{x}^{k}\right) \\
& =\left(x^{k}-\tilde{x}^{k}\right)^{\top}\left((1+\mu) x-\left(\mu x^{k}+\tilde{x}^{k}\right)\right),
\end{aligned}
$$

and thus (4.3) holds. Similarly, we can prove (4.4) and the proof is complete. 
The following result can be proved by similar arguments to those in $[11,14,15]$. Hence, the proof is omitted.

Theorem $4.3([11,14,15])$. If $\inf _{k=0}^{\infty} \beta_{k}:=\beta>0$, then the sequence $\left\{u^{k}\right\}$ which is generated by the proposed method converges to some $\mathrm{u}^{\infty}$ that is a solution of $\mathrm{VI}(\Omega, \mathrm{F})$.

\section{Convergence rate}

Recall that $\Omega^{*}$ can be characterized as (see [10, (2.3.2)])

$$
\Omega^{*}=\bigcap_{\mathfrak{u} \in \Omega}\left\{\tilde{u} \in \Omega:(u-\tilde{u})^{\top} F(u) \geqslant 0\right\} .
$$

This implies that $\tilde{u} \in \Omega$ is an approximate solution of $\operatorname{VI}(\Omega, F)$ with accuracy $\epsilon>0$ if it satisfies

$$
(\tilde{u}-u)^{\top} F(u) \leqslant \epsilon, \quad \forall u \in \Omega .
$$

Now, we show that after $t$ iterations of the proposed method, we can find a $\tilde{u} \in \Omega$ such that (5.1) is satisfied with $\epsilon=\mathrm{O}(1 / \mathrm{t})$.

Lemma 5.1. Let $\tilde{u}^{k}$ be generated by (2.1) and $\mathrm{u}^{k+1}\left(\gamma \alpha_{k}\right)$ be generated by (2.4). Then we have the following

$$
\left(u^{k+1}\left(\gamma \alpha_{k}\right)-\tilde{u}^{k}\right)^{\top} \beta_{k} F\left(\tilde{u}^{k}\right) \geqslant \alpha_{k}^{*}\left\|d\left(u^{k}, \tilde{u}^{k}\right)\right\|_{G}^{2}+\left(u^{k+1}\left(\gamma \alpha_{k}\right)-u^{k}\right)^{\top} G d\left(u^{k}, \tilde{u}^{k}\right) .
$$

Proof. It follows from (3.8) that

$$
\begin{aligned}
\left(u^{k+1}\left(\gamma \alpha_{k}\right)-\tilde{u}^{k}\right)^{\top} \beta_{k} F\left(\tilde{u}^{k}\right) \geqslant & \left(u^{k+1}\left(\gamma \alpha_{k}\right)-\tilde{u}^{k}\right)^{\top} G d\left(u^{k}, \tilde{u}^{k}\right)-\mu\left\|x^{k}-\tilde{x}^{k}\right\|^{2}-\frac{v_{k} \mu}{2}\left\|y^{k}-\tilde{y}^{k}\right\|^{2} \\
= & \left(u^{k+1}\left(\gamma \alpha_{k}\right)-u^{k}+u^{k}-\tilde{u}^{k}\right)^{\top} G d\left(u^{k}, \tilde{u}^{k}\right)-\mu\left\|x^{k}-\tilde{x}^{k}\right\|^{2} \\
& -\frac{v_{k} \mu}{2}\left\|y^{k}-\tilde{y}^{k}\right\|^{2} \\
= & \left\|u^{k}-\tilde{u}^{k}\right\|_{G}^{2}-\mu\left\|x^{k}-\tilde{x}^{k}\right\|^{2}-\frac{v_{k} \mu}{2}\left\|y^{k}-\tilde{y}^{k}\right\|^{2}+\left(u^{k}-\tilde{u}^{k}\right)^{\top} \xi^{k} \\
& +\left(u^{k+1}\left(\gamma \alpha_{k}\right)-u^{k}\right)^{\top} G d\left(u^{k}, \tilde{u}^{k}\right) \\
= & \varphi\left(u^{k}, \tilde{u}^{k}\right)+\left(u^{k+1}\left(\gamma \alpha_{k}\right)-u^{k}\right)^{\top} G d\left(u^{k}, \tilde{u}^{k}\right) \\
= & \alpha_{k}^{*}\left\|d\left(u^{k}, \tilde{u}^{k}\right)\right\|_{G}^{2}+\left(u^{k+1}\left(\gamma \alpha_{k}\right)-u^{k}\right)^{\top} G d\left(u^{k}, \tilde{u}^{k}\right) .
\end{aligned}
$$

Lemma 5.2. Given $\mathrm{u}^{\mathrm{k}} \in \mathbb{R}_{++}^{\mathrm{n}} \times \mathbb{R}_{++}^{\mathrm{m}}$. Let $\tilde{\mathrm{u}}^{\mathrm{k}}$ be generated by (2.1) and $\mathrm{u}^{\mathrm{k}+1}\left(\gamma \alpha_{\mathrm{k}}\right)$ be generated by (2.4). If we take $\tau_{\mathrm{k}}=\frac{1-\mu}{1+\mu} \gamma \alpha_{\mathrm{k}}$, then for any $\mathrm{u} \in \Omega$, we have

$$
\left(u-\tilde{u}^{k}\right)^{\top} \beta_{k} F(u) \geqslant \frac{(2-\gamma) \alpha_{k}}{2}\left\|d\left(u^{k}, \tilde{u}^{k}\right)\right\|_{G}^{2}+\frac{1}{2 \tau_{k}}\left(\left\|u^{k+1}\left(\gamma \alpha_{k}\right)-u\right\|_{G}^{2}-\left\|u^{k}-u\right\|_{G}^{2}\right) .
$$

Proof. It follows from (3.2)

$$
\left(u-u^{k+1}\left(\gamma \alpha_{k}\right)\right)^{\top} \beta_{k} F\left(\tilde{u}^{k}\right) \geqslant \frac{1}{2 \tau_{k}}\left(\left\|u^{k+1}\left(\gamma \alpha_{k}\right)-u\right\|_{G}^{2}-\left\|u^{k}-u\right\|_{G}^{2}\right)+\frac{1-\mu}{2 \tau_{k}(1+\mu)}\left\|u^{k}-u^{k+1}\left(\gamma \alpha_{k}\right)\right\|_{G}^{2} .
$$

Adding (5.2) and (5.4), we obtain

$$
\begin{aligned}
\left(u-\tilde{u}^{k}\right)^{\top} \beta_{k} F\left(\tilde{u}^{k}\right) \geqslant & \alpha_{k}\left\|d\left(u^{k}, \tilde{u}^{k}\right)\right\|_{G}^{2}+\left(u^{k+1}\left(\gamma \alpha_{k}\right)-u^{k}\right)^{\top} G d\left(u^{k}, \tilde{u}^{k}\right) \\
& +\frac{1}{2 \tau_{k}}\left(\left\|u^{k+1}\left(\gamma \alpha_{k}\right)-u\right\|_{G}^{2}-\left\|u^{k}-u\right\|_{G}^{2}\right)+\frac{1-\mu}{2 \tau_{k}(1+\mu)}\left\|u^{k}-u^{k+1}\left(\gamma \alpha_{k}\right)\right\|_{G}^{2} .
\end{aligned}
$$


Using the following inequality

$$
\left(u^{k+1}\left(\gamma \alpha_{k}\right)-u^{k}\right)^{\top} G d\left(u^{k}, \tilde{u}^{k}\right) \geqslant-\frac{1-\mu}{2 \tau_{k}(1+\mu)}\left\|u^{k}-u^{k+1}\left(\gamma \alpha_{k}\right)\right\|_{G}^{2}-\frac{\tau_{k}(1+\mu)}{2(1-\mu)}\left\|d\left(u^{k}, \tilde{u}^{k}\right)\right\|_{G}^{2},
$$

we obtain

$$
\left(u-\tilde{u}^{k}\right)^{\top} \beta_{k} F\left(\tilde{u}^{k}\right) \geqslant \frac{(2-\gamma) \alpha_{k}}{2}\left\|d\left(u^{k}, \tilde{u}^{k}\right)\right\|_{G}^{2}+\frac{1}{2 \tau_{k}}\left(\left\|u^{k+1}-u\right\|_{G}^{2}-\left\|u^{k}-u\right\|_{G}^{2}\right),
$$

and by using the monotonicity of $F$, we obtain (5.3).

Now, we are ready to present the $\mathrm{O}(1 / \mathrm{t})$ convergence rate of the proposed method.

Theorem 5.3. For any integer $\mathrm{t}>0$, we have $a \tilde{u}_{t} \in \Omega$ that satisfies

$$
\left(\tilde{u}_{t}-u\right)^{\top} F(u) \leqslant \frac{1}{2 \gamma_{t}}\left\|u-u^{0}\right\|_{G}^{2}, \quad \forall u \in \Omega,
$$

where

$$
\tilde{u}_{t}=\frac{1}{\gamma_{t}} \sum_{k=0}^{t} \beta_{k} \tau_{k} \tilde{u}^{k} \text { and } \gamma_{t}=\sum_{k=0}^{t} \beta_{k} \tau_{k} .
$$

Proof. Summing the inequality (5.3) over $k=0, \cdots, t$, we obtain

$$
\left(\left(\sum_{k=0}^{t} \beta_{k} \tau_{k}\right) u-\sum_{k=0}^{t} \beta_{k} \tau_{k} \tilde{u}^{k}\right)^{\top} F(u)+\frac{1}{2}\left\|u-u^{0}\right\|_{G}^{2} \geqslant 0 .
$$

Using the notations of $\Upsilon_{t}$ and $\tilde{u}_{t}$ in the above inequality, we derive

$$
\left(\tilde{u}_{t}-u\right)^{\top} F(u) \leqslant \frac{1}{2 \gamma_{t}}\left\|u-u^{0}\right\|_{G}^{2}, \quad \forall u \in \Omega .
$$

Indeed, $\tilde{u}_{t} \in \Omega$ because it is a convex combination of $\tilde{u}^{0}, \tilde{u}^{1}, \ldots, \tilde{u}^{t}$. The proof is complete.

It follows from (3.15) that

$$
\Upsilon_{t} \geqslant \frac{(1-\mu) \beta \gamma}{2(1+\mu)}(t+1)
$$

Suppose that for any compact set $\mathcal{D} \subset \mathbb{Z}$, let $d=\sup \left\{\left\|u-u^{0}\right\|_{G} \mid z \in \mathcal{D}\right\}$. For any given $\epsilon>0$, after at most

$$
t=\left[\frac{(1+\mu) d^{2}}{(1-\mu) \beta \gamma \epsilon}-1\right]
$$

iterations, we have

$$
\left(\tilde{u}_{\mathfrak{t}}-\mathfrak{u}\right)^{\top} \mathrm{F}(\mathrm{u}) \leqslant \epsilon, \quad \forall \mathrm{u} \in \mathcal{D} .
$$

That is, the $\mathrm{O}(1 / \mathrm{t})$ convergence rate of the inexact LQP-SQP ADM is established in an ergodic sense.

\section{Numerical experiments}

We apply our proposed method in the following examples to illustrate its advantage and efficiency.

\subsection{Numerical experiments for traffic equilibrium problems}

The test examples in this section arise from the traffic equilibrium problems.

\subsubsection{Traffic equilibrium problems}

We consider a network [15] shown in Figure 1 which consists of 25 nodes, 37 links and 6 origin/destination (O/D) pairs. We apply the proposed method in this traffic network equilibrium problems with two modifications, one with link capacities and the other with both link capacities and low bounds on travel demands. We use the 
same notation as in [15]. The traffic equilibrium problems can be described as follows:

$$
\left(x-x^{*}\right)^{\top} F\left(x^{*}\right) \geqslant 0, \quad \forall x \in S,
$$

where

$$
F(x)=A t\left(A^{\top} x\right)-B \lambda\left(B^{\top} x\right) .
$$

For practical applications, $S$ has the following different forms.

- Traffic equilibrium problems with link capacity bound,

$$
S=\left\{x \in \mathbb{R}^{n} \mid A^{\top} x \leqslant b, x \geqslant 0\right\},
$$

$\mathrm{b}$ is the given link capacity vector

- Traffic equilibrium problems with link capacity bound and demand lower bound,

$$
S=\left\{x \in \mathbb{R}^{\mathfrak{n}} \mid A^{\top} x \leqslant b, B^{\top} x \geqslant d, x \geqslant 0\right\} .
$$

It is clear that all these traffic equilibrium problems are special cases of the structured variational inequality (1.1)(1.2). We apply the proposed method to solve these problems.

In all test implementations, we use the forms of function $t(f)$ and $\lambda(d)$ from [15], and we take $u^{0}=\left(x^{0}, y^{0}\right)$, where each element of $x^{0}$ and $y^{0}$ is equal to $1, \mu=0.01, \gamma=1.95$, and $\eta=0.95$. For this test problem, the stopping criterion

for different $\varepsilon$ are reasonable.

$$
\max \left\{\frac{\left\|e_{x}\left(u^{k}\right)\right\|_{\infty}}{\left\|e_{x}\left(u^{0}\right)\right\|_{\infty}},\left\|e_{y}\left(u^{k}\right)\right\|_{\infty}\right\} \leqslant \varepsilon,
$$

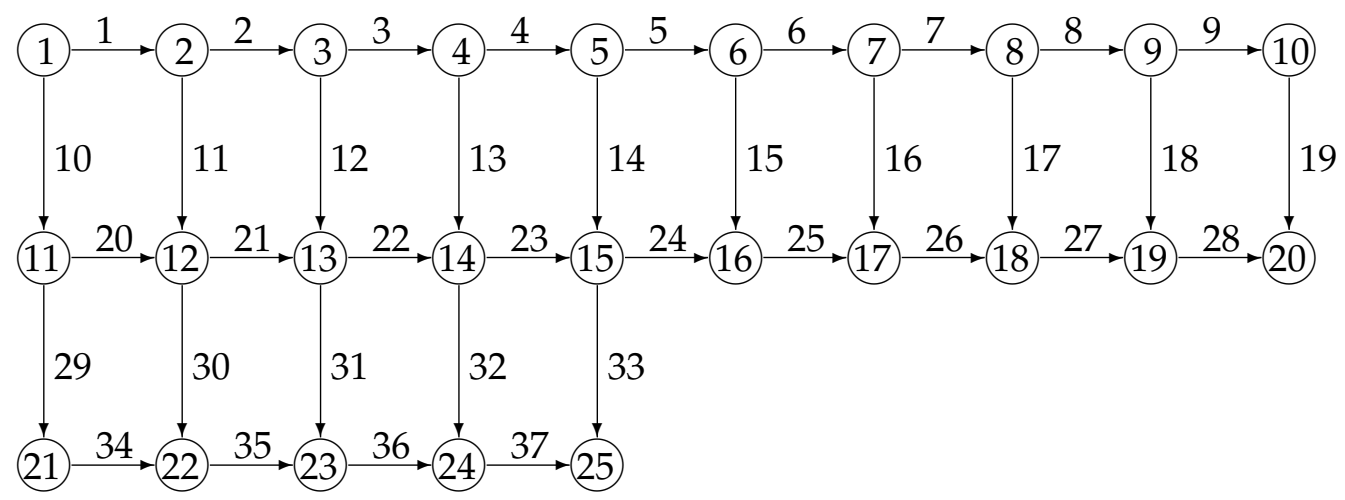

Figure 1: A directed network with 25 nodes and 37 links.

\subsubsection{Problems with link capacity bounds}

The constraints set of problems with link capacity bounds is $S=\left\{x \in \mathbb{R}^{n} \mid A^{\top} x \leqslant b, x \geqslant 0\right\}$, where $b$ is a given capacity vector. We report the numbers of iteration, the number of function evaluations, and the CPU time for different capacities and different $\varepsilon$ in Tables 1 and 2 .

Table 1: Numerical results for different $\varepsilon$ with $b=40$.

\begin{tabular}{|c||c|c|c||c|c|c||c|c|c|}
\hline \multicolumn{1}{|c||}{$\begin{array}{c}\text { Different } \\
\varepsilon\end{array}$} & \multicolumn{3}{c||}{ The method in [11] } & \multicolumn{2}{c|}{ The method in [15] } & \multicolumn{2}{c|}{ The proposed method } \\
\cline { 2 - 10 } & $\mathrm{k}$ & 1 & $\mathrm{CPU}(\mathrm{s})$ & $\mathrm{k}$ & $\mathrm{l}$ & $\mathrm{CPU}(\mathrm{s})$ & $\mathrm{k}$ & $\mathrm{l}$ & $\mathrm{CPU}(\mathrm{s})$ \\
\hline $10^{-5}$ & 209 & 450 & 0.068 & 217 & 457 & 0.068 & 202 & 424 & 0.048 \\
\hline $10^{-6}$ & 249 & 534 & 0.068 & 247 & 522 & 0.063 & 238 & 496 & 0.061 \\
\hline $10^{-7}$ & 279 & 596 & 0.79 & 275 & 583 & 0.077 & 270 & 560 & 0.075 \\
\hline
\end{tabular}

As illustrated in Subsection 6.1.1, the output vector $x$ is the path-flow, and the link flow vector is $A^{\top} x$. In fact, $\mathrm{y}^{*}$ in the output is referred to as the toll charge on the congested link. For the example with link capacity $b=40$ we list the optimal link flow and the toll charge in Table 3. Indeed, the link toll charge is greater than zero if and only if the link flow reaches capacity. 
Table 2: Numerical results for different $\varepsilon$ with $b=50$.

\begin{tabular}{|c||c|c|c||c|c|c||c|c|c|}
\hline \multicolumn{1}{|c||}{ Different } & \multicolumn{3}{c||}{ The method in [11] } & \multicolumn{3}{c||}{ The method in [15] } & \multicolumn{3}{c|}{ The proposed method } \\
\cline { 2 - 10 }$\varepsilon$ & $\mathrm{k}$ & $\mathrm{l}$ & $\mathrm{CPU}(\mathrm{s})$ & $\mathrm{k}$ & $\mathrm{l}$ & $\mathrm{CPU}(\mathrm{s})$ & $\mathrm{k}$ & $\mathrm{l}$ & $\mathrm{CPU}(\mathrm{s})$ \\
\hline $10^{-5}$ & 403 & 833 & 0.11 & 405 & 853 & 0.094 & 391 & 795 & 0.086 \\
\hline $10^{-6}$ & 485 & 997 & 0.11 & 488 & 1021 & 0.094 & 475 & 963 & 0.10 \\
\hline $10^{-7}$ & 567 & 1161 & 0.14 & 570 & 1185 & 0.011 & 560 & 1133 & 0.11 \\
\hline
\end{tabular}

Table 3: The optimal link flow and the toll charge on the link when $b=40$.

\begin{tabular}{c|r|r||r|r|r||r|r|r||r|r|r}
\hline Link & Flow & Charge & Link & Flow & Charge & Link & Flow & Charge & Link & Flow & Charge \\
\hline 1 & 40.00 & 4.3 & 11 & 1.85 & 0 & 21 & 40.00 & 1.1 & 31 & 11.96 & 0 \\
2 & 38.15 & 0 & 12 & 11.96 & 0 & 22 & 40.00 & 136.6 & 32 & 40.00 & 164.2 \\
3 & 40.00 & 163.2 & 13 & 26.19 & 0 & 23 & 26.19 & 0 & 33 & 40.00 & 135.6 \\
4 & 13.81 & 0 & 14 & 13.81 & 0 & 24 & 0 & 0 & 34 & 26.19 & 0 \\
5 & 0 & 0 & 15 & 0 & 0 & 25 & 0 & 0 & 35 & 28.04 & 0 \\
6 & 0 & 0 & 16 & 0 & 0 & 26 & 0 & 0 & 36 & 40.00 & 301.3 \\
7 & 0 & 0 & 17 & 0 & 0 & 27 & 0 & 0 & 37 & 0 & 0 \\
8 & 0 & 0 & 18 & 0 & 0 & 28 & 0 & 0 & - & - & - \\
9 & 0 & 0 & 19 & 0 & 0 & 29 & 26.19 & 0 & - & - & - \\
10 & 40.00 & 1.1 & 20 & 40.00 & 1.8 & 30 & 1.85 & 0 & - & - & - \\
\hline
\end{tabular}

\subsubsection{Problems with link capacity bounds and demand low bounds}

The constraints set of problem in this subsection is $S=\left\{x \in \mathbb{R}^{n} \mid A^{\top} x \leqslant b, B^{\top} x \geqslant d, x \geqslant 0\right\}$, where $b$ and $d$ are given vectors. In the test example we let each element of $b$ and $d$ be equal to 40 and 10, respectively. We report the numbers of iterations, the mapping evaluations, and the CPU time for different $\varepsilon$ in Table 4.

Table 4: Numerical results for different $\varepsilon$ with link flow capacity $A^{\top} x \leqslant 40$ and demand low bound $B^{\top} x \geqslant 10$.

\begin{tabular}{|c||c|c|c||c|c|c||c|c|c|}
\hline \multicolumn{1}{|c||}{$\begin{array}{c}\text { Different } \\
\varepsilon\end{array}$} & \multicolumn{3}{c||}{ The method in [11] } & \multicolumn{3}{c||}{ The method in [15] } & \multicolumn{3}{c|}{ The proposed method } \\
\cline { 2 - 10 } & $\mathrm{k}$ & $\mathrm{l}$ & $\mathrm{CPU}(\mathrm{s})$ & $\mathrm{k}$ & $\mathrm{l}$ & $\mathrm{CPU}(\mathrm{s})$ & $\mathrm{k}$ & $\mathrm{l}$ & CPU (s) \\
\hline $10^{-5}$ & 400 & 850 & 0.11 & 392 & 834 & 0.089 & 257 & 550 & 0.07 \\
\hline $10^{-6}$ & 485 & 1028 & 0.12 & 470 & 998 & 0.09 & 315 & 670 & 0.081 \\
\hline $10^{-7}$ & 571 & 1209 & 0.13 & 561 & 1187 & 0.011 & 375 & 795 & 0.1 \\
\hline
\end{tabular}

The dual variable $y^{*}$ can be divided into two subvectors $y_{I}^{*}$ (to the capacity constraints $A^{\top} x \leqslant b$ ) and $y_{I I}^{*}$ (to the demand lower bounds $B^{\top} x \geqslant d$ ). Here $y_{I}^{*}$ in the output is referred to as the toll charge on the congested link, while $y_{\text {II }}^{*}$ represents the subsidy on the O/D pair. For the test example, we list the optimal link flow and the toll charge in Table 5. The optimal demand and the subsidy of each O/D pair are given in Table 6. The outputs coincide with the optimal condition.

Table 5: The optimal link flow and the toll charge on the link when $b=40$.

\begin{tabular}{c|r|r||r|r|r||r|r|r||r|r|r}
\hline Link & Flow & Charge & Link & Flow & Charge & Link & Flow & Charge & Link & Flow & Charge \\
\hline 1 & 40.00 & 17.4 & 11 & 10.00 & 0 & 21 & 40.00 & 6.9 & 31 & 10.00 & 0 \\
2 & 40.00 & 12.6 & 12 & 10.00 & 0 & 22 & 40.00 & 306.5 & 32 & 34.65 & 0 \\
3 & 40.00 & 333.9 & 13 & 14.61 & 0 & 23 & 19.96 & 0 & 33 & 25.35 & 0 \\
4 & 25.39 & 0 & 14 & 8.69 & 0 & 24 & 3.30 & 0 & 34 & 23.14 & 0 \\
5 & 16.70 & 0 & 15 & 9.88 & 0 & 25 & 13.18 & 0 & 35 & 30.00 & 0 \\
6 & 6.82 & 0 & 16 & 0 & 0 & 26 & 13.18 & 0 & 36 & 40.00 & 294.0 \\
7 & 6.82 & 0 & 17 & 0 & 0 & 27 & 13.18 & 0 & 37 & 14.65 & 0 \\
8 & 6.82 & 0 & 18 & 6.82 & 0 & 28 & 20.00 & 0 & - & - & - \\
9 & 0 & 0 & 19 & 0 & 0 & 29 & 23.14 & 0 & - & - & - \\
10 & 40.00 & 25.6 & 20 & 36.86 & 0 & 30 & 6.86 & 0 & - & - & - \\
\hline
\end{tabular}

Table 6: The optimal demand and the related subsidy.

\begin{tabular}{|c||c|c|c|c|c|c|}
\hline (O,D) Pair & $(1,20)$ & $(1,25)$ & $(2,20)$ & $(3,25)$ & $(1,24)$ & $(11,25)$ \\
\hline Optimal demand & 10 & 10 & 10 & 10 & 60 & 20 \\
\hline Subsidy & 909.4 & 730.6 & 710.2 & 29.1 & 0 & 0 \\
\hline
\end{tabular}

Tables 1, 2, and 4 show that the proposed method solves the traffic equilibrium problem very efficiently. 


\section{Conclusions}

In this paper, we have proposed an inexact LQP-SQP ADM for solving structured variational inequalities. Each iteration of the LQP-SQP ADM includes prediction and correction steps where prediction and correction points are obtained by solving series of related systems of nonlinear equations. Global convergence of the proposed method is proved under mild assumptions. Some preliminary numerical results are reported to verify the effectiveness of the proposed LQP-SQP ADM in practice.

\section{Acknowledgment}

This work was supported by the Deanship of Scientific Research (DSR), King Abdulaziz University, Jeddah, under grant No. (D-012-130-1438). The authors, therefore, gratefully acknowledge the DSR technical and financial support. Finally, the authors thank the reviewer for comments/suggestions.

\section{References}

[1] A. E. Al-Mazrooei, A. Latif, J. C. Yao, Solving generalized mixed equilibria, variational inequalities, and constrained convex minimization, Abstr. Appl. Anal., 2014 (2014), 26 pages. 1

[2] A. Auslender, M. Teboulle, S. Ben-Tiba, A logarithmic-quadratic proximal method for variational inequalities, Computational optimization-a tribute to Olvi Mangasarian, Part I, Comput. Optim. Appl., 12 (1999), 31-40. 2

[3] A. Bnouhachem, On LQP alternating direction method for solving variational inequality problems with separable structure, J. Inequal. Appl., 2014 (2014), 15 pages. 1

[4] A. Bnouhachem, Q. H. Ansari, A descent LQP alternating direction method for solving variational inequality problems with separable structure, Appl. Math. Comput., 246 (2014), 519-532.

[5] A. Bnouhachem, H. Benazza, M. Khalfaoui, An inexact alternating direction method for solving a class of structured variational inequalities, Appl. Math. Comput., 219 (2013), 7837-7846. 1

[6] A. Bnouhachem, A. Hamdi, Parallel LQP alternating direction method for solving variational inequality problems with separable structure, J. Inequal. Appl., 2014 (2014), 14 pages.

[7] A. Bnouhachem, A. Latif, Q. H. Ansari, On the $\mathrm{O}(1 / \mathrm{t})$ convergence rate of the alternating direction method with LQP regularization for solving structured variational inequality problems, J. Inequal. Appl., 2016 (2016), 14 pages.

[8] A. Bnouhachem, M. H. Xu, An inexact LQP alternating direction method for solving a class of structured variational inequalities, Comput. Math. Appl., 67 (2014), 671-680. 1

[9] L.-C. Ceng, A. Latif, J.-C. Yao, On solutions of a system of variational inequalities and fixed point problems in Banach spaces, Fixed Point Theory Appl., 2013 (2013), 34 pages. 1

[10] F. Facchinei, J.-S. Pang, Finite-dimensional variational inequalities and complementarity problems, Vol. I and II, Springer Series in Operations Research, Springer-Verlag, New York, (2003). 5

[11] X.-L. Fu, A. Bnouhachem, An self-adaptive LQP method for constrained variational inequalities, Appl. Math. Comput., 189 (2007), 1586-1600. 1, 4, 4.3, 1, 2, 4

[12] R. Glowinski, Numerical methods for nonlinear variational problems, Springer Series in Computational Physics, Springer-Verlag, New York, (1984). 1

[13] R. Glowinski, P. Le Tallec, Augmented Lagrangian and operator-splitting methods in nonlinear mechanics, SIAM Studies in Applied Mathematics, Society for Industrial and Applied Mathematics (SIAM), Philadelphia, PA, (1989). 1

[14] H.-J. He, K. Wang, X.-J. Cai, D.-R. Han, An LQP-based two-step method for structured variational inequalities, J. Oper. Res. Soc. China, 5 (2017), 301-317. 1, 4, 4.3

[15] B.-S. He, Y. Xu, X.-M. Yuan, A logarithmic-quadratic proximal prediction-correction method for structured monotone variational inequalities, Comput. Optim. Appl., 35 (2006), 19-46. 1, 4, 4.3, 6.1.1, 1, 2, 4

[16] B.-S. He, H. Yang, S.-L. Wang, Alternating direction method with self-adaptive penalty parameters for monotone variational inequalities, J. Optim. Theory Appl., 106 (2000), 337-356. 1

[17] B.-S. He, J. Zhou, A modified alternating direction method for convex minimization problems, App. Math. Lett., 13 (2000), 123-130.

[18] Z.-K. Jiang, A. Bnouhachem, A projection-based prediction-correction method for structured monotone variational inequalities, Appl. Math. Comput., 202 (2008), 747-759.

[19] S. Kontogiorgis, R. R. Meyer, A variable-penalty alternating directions method for convex optimization, Math. Programming, 83 (1998), 29-53.

[20] A. Latif, L.-C. Ceng, Q. H. Ansari, Multi-step hybrid viscosity method for systems of variational inequalities defined over sets of solutions of an equilibrium problem and fixed point problems, Fixed Point Theory Appl., 2012 (2012), 26 pages.

[21] A. Latif, D. T. Luc, Variational relation problems: existence of solutions and fixed points of contraction mappings, Fixed Point Theory Appl., 2013 (2013), 10 pages. 1

[22] M. Li, A hybrid LQP-based method for structured variational inequalities, Int. J. Comput. Math., 89 (2012), $1412-1425$. 1 
[23] M. Tao, X.-M. Yuan, On the $\mathrm{O}(1 / \mathrm{t})$ convergence rate of alternating direction method with logarithmic-quadratic proximal regularization, SIAM J. Optim., 22 (2012), 1431-1448. 1

[24] M. Teboulle, Convergence of proximal-like algorithms, SIAM J. Optim., 7 (1997), 1069-1083. 1

[25] X.-M. Yuan, M. Li, An LQP-based decomposition method for solving a class of variational inequalities, SIAM J. Optim., 21 (2011), 1309-1318. 1, 1 